\title{
Immunizing Vulnerable Populations Like Rag Pickers, Garbage Collectors, Municipality Workers and Newspaper Hawkers against Rabies in Shimla Municipality, HP, India
}

\author{
Omesh Kumar Bharti \\ Corporation Health Officer, Municipal Corporation, Shimla, India \\ Email: bhartiomesh@yahoo.com
}

Received 30 December 2014; accepted 16 January 2015; published 20 January 2015

Copyright (C) 2015 by authors and Scientific Research Publishing Inc.

This work is licensed under the Creative Commons Attribution International License (CC BY). http://creativecommons.org/licenses/by/4.0/

(c) (i) Open Access

\begin{abstract}
Background: Rabies is a zoonotic disease and many vulnerable sections like rag pickers and municipality workers neglect animal bites due to ignorance of their potential deadly outcomes. Stray dogs abound in garbage pits and this population is exposed to their attacks. It should be a mandate for municipalities to help protect their sanitary workforce, especially rag pickers, from deadly infectious diseases such as Rabies, Hepatitis-B, HIV, Tetanus etc. Objectives: Objective of this study was to study methods to provide pre-exposure Rabies vaccination for such highly exposed populations by engaging them and understanding their perception of this disease through a constant dialogue with them. Methods: We started by engaging with the rag pickers to know how best to entice them to get themselves immunized. We then attempted to search literature for the most practical methods likely to succeed in reducing risk of rabies deaths in this population. Results: WHO approved 3 injections of $0.1 \mathrm{ml}$ tissue culture vaccine on days 0,7 and 21 were tried but were shown to result in many dropouts among rag pickers for repeat injections. We then followed a method where $0.1 \mathrm{ml}$ of rabies vaccine was injected at 4 different anatomical sited in one setting. This proved acceptable and relatively inexpensive. A small number of subjects were studied by determination of neutralizing antibody by RFFIT, which proved immunogenic having anamnestic response on boosters given single IM or at 4 sites ID subsequently, implying that short schedule rabies pre-exposure vaccination can be done in high risk groups and may save lives if applied to the poorest that are highly exposed.
\end{abstract}

\section{Keywords}

Pre-Exposure Rabies Vaccination, Intradermal Antirabies Vaccination (IDRV), Rag Pickers 


\section{Introduction}

Rabies is a zoonotic disease and many vulnerable sections neglect animal bites due to ignorance of their deadly outcome. Poverty and illiteracy are also contributing factors for not seeking any medical assistance in emergencies like dog bites as they admitted to having been bitten many times by stray dogs and monkeys but did not realize the urgency of seeking any medical care.

It is the mandate of any municipality to safeguard the health of its sanitary workforce, especially rag pickers, from the diseases they are vulnerable to like Rabies, Hepatitis-B and C, HIV, Tetanus etc. [1]. The problem is that rag pickers and garbage collectors are not owned by any municipality despite them being important in keeping the cities clean and green without any burden on municipalities. It has been observed that the majority of rag pickers are not available for vaccination next time. WHO schedule of pre-vaccination against rabies at day 0,7 and 21/28, which is a gold standard, is not possible in such a vulnerable populations, which need special protocols for pre-exposure vaccination to protect them from rabies. Therefore they need to be vaccinated as much as possible at the very first contact with them. A detailed literature review was done to know the ways to vaccinate such vulnerable populations for best outcomes against rabies.

\section{Literature Review}

We did not find any study of pre exposure prophylaxis to rag pickers with rabies vaccine. There were some studies that relate to travelers to endemic countries. One of the studies by Pakamatz Khawplod et al., proves that $0.1 \mathrm{ml}$ of a WHO-accepted tissue culture rabies vaccine injected at two ID sites (both deltoid regions) conferred immune memory for at least1 year. Two booster injections 1 year later resulted in an accelerated immune response indicating that immunoglobulin may not be necessary if such a subject experiences a rabies exposure within 1 year [2]. Another study by the same author stresses that even one clinic visit with two-site $0.1 \mathrm{ml}$ ID injections or a one-site single $0.1 \mathrm{ml}$ pre-exposure rabies vaccination is enough to prime the host immune memory for at least one to three years [3]. Following a small study by Turner, 4-site ID injection on the limbs was recommended for emergency pre-exposure use in the UK and was put into practice in some travel clinics [4]. Multiple-site ID doses on one day is better than nothing, and RIGs (Rabies Immunoglobulins) is often not available in remote parts which is not required in previously vaccinated people who have memory cells in active stage.

\section{Method}

We get 8 - 10 new patients in our clinic everyday bitten by dogs and monkeys but we rarely see rag pickers or such populations seeking post-exposure prophylaxis. This provoked us to think of giving them pre exposure prophylaxis on World Rabies Day (WRD) in the year 2010. A wide publicity in media through press statements was done and also messages were given to the rag picker groups, municipal officials and employers of newspaper hawkers in Shimla town for pre-exposure prophylaxis with rabies vaccine on World Rabies Day (WRD) on September 28, 2010. At the very outset in 2010 we gave 2 site rabies vaccine, $0.1 \mathrm{ml}$. each site on deltoids to 225 persons who came for getting vaccination. Next time on day 7 only 45 turned up and then on day 21/28 only 16 turned up for getting vaccination. Vaccine used was Rabipur that was left over from hospital stocks i.e. 0.2 $\mathrm{ml}$ from each vial after distribution among four patients [5].

To get a better compliance next time on WRD 2012, we went to rag pickers on the municipality garbage dumping sites. We counseled many of them on spot and thereafter immunized 57 new rag pickers onsite with $0.1 \mathrm{ml}$ IDRV on four sites to be followed later on day 7 and 21. But next time only 13 were available and out of them 4 refused to get immunization for fear of pain and injection. On day 21, none was available as they had left for their homes in other states of the country. Some municipality workers and door to door garbage collectors, who were available, were also immunized. Vaccine used was Vaxirab-N.

Learning a lesson from the high dropout of such vulnerable populations, this time in the year 2014, we started a long campaign in May 2014 to immunize maximum of them with one time 4 site $0.1 \mathrm{ml}$ ID vaccination. Against the target of 1000 workers/garbage collectors we could rope in a total of 412 of them in four months period till this WRD 2014. We interviewed some of them if they remembered their last vaccination status and blood samples were taken for Rapid Fluorescent Focus Inhibition test (RFFIT) to check the immunogenicity. Vaccine used was Vaxirab-N and Abhayrab.

To get them further engaged, we organized a five day free health insurance camp for Rastriya Swastheya Bi- 
ma Yojna (RSBY), an insurance scheme of Government of India for sanitary workers. A meager sum of Rs. 30 $(<1 \$)$ for each family card was paid by the SUNYA project of European Union and Municipal Corporation. A total of 288 families (about 1000 people) of garbage collectors and rag pickers got enrolled for this insurance scheme and 126 of them were vaccinated on the spot with rabies vaccine at four sites and inj. TT, some children were given DPT, TT and Hepatitis-B as well who came with their parents for enrolment.

\section{Results and Discussion}

WHO approved 3 injection of $0.1 \mathrm{ml}$ tissue culture vaccine on days 0,7 and 21were tried but were shown to result in many dropouts among rag pickers for the repeat injections. We then followed a method where $0.1 \mathrm{ml}$ of rabies vaccine was to be injected at 4 different anatomical sited in one setting. This proved acceptable and relatively inexpensive. A small number of subjects were studied by determination of neutralizing antibody by RFFIT which proved immunogenicity of four site $0.1 \mathrm{ml}$ dose of rabies pre-exposure vaccination given ID having anamnestic response on boosters given on 4 sites ID subsequently.

Blood samples were collected at day 0 from seven sanitary workers who could recollect their previous vaccination status before 4 site vaccination in 2014 and consented for blood samples. These had antibody titers (RFFIT) ranged from $2.5 \mathrm{IU} / \mathrm{ml}-6.5 \mathrm{IU} / \mathrm{ml}$. The four municipal sanitary workers who could recollect that they were vaccinated in 2012 at 4 sites ID had antibody titers 2.5, 3.5, 3.5 and $4.5 \mathrm{IU} / \mathrm{ml}$ respectably with or without a single booster injection given subsequently in-between. All the four were never immunized before 2012. That corroborated our findings for having rabies antibodies titers for at least two years as in the studies by Pakamatz Khawplod et al. referred above.

The other two were never vaccinated but still had titers because they are exposure to the work of lifting live (for animal birth control, ABC) as well as dead (for disposal) animals (Dogs/Monkeys etc.) reported to the corporation and may have acquired immunity because of that as is proved in many such conditions like handling and butchering dogs in African countries like Nigeria [6] and pose serious risk to such people by such animals [7]-[9] as $22.6 \%$ of apparently healthy dogs were found to be positive for rabies virus in Nigeria [10] [11]. This poses serious risk to dog handlers of municipalities and therefore urgent need for pre exposure rabies vaccination.

Table 1 shows the exact history of vaccination of these sanitary workers. This also shows an accelerated immune response to single boosters at different intervals. One worker (MC 75), who did not get any booster in-between after 4 site IDRV in 2012 and who was given IDRV at 4 sites $0.1 \mathrm{ml}$ ID after 20 months, and blood sample was taken on day 10 showed a very good protective antibody levels, especially if we compare this with that of MC 55 who was in similar position except a booster at 20 months. Early booster on day 7 (MC 58) and late booster at 20 months (MC 75) had comparable effect in generating antibodies. MC 104 was given single IM shots every year for three years, as he worked with ABC programme of corporation and had good titers after five years.

\section{Conclusions}

One time four site $0.1 \mathrm{ml}$ ID pre-exposure prophylaxis against rabies is immunogenic and is a better alternate to

Table 1. Antibody Titers (RFFIT)-Vaccine used in 2014 is Vaxirab-N potency 7.18 IU/ Vial, Batch CM-132, vaccine Abhayrab was also used in some cases.

\begin{tabular}{|c|c|c|c|c|c|}
\hline Code & $\begin{array}{l}\text { Date of first } \\
\text { vaccination }\end{array}$ & $\begin{array}{l}\text { Vaccination type on first } \\
\text { vaccination }\end{array}$ & $\begin{array}{l}\text { Date of last } \\
\text { vaccination }\end{array}$ & $\begin{array}{l}\text { Last Vaccination type } \\
\text { (Boosters on D/M) }\end{array}$ & $\begin{array}{l}\text { RFFIT titers at } 20 \text { Months, before } 4 \\
\text { shot IDRV in } 2014 \text { as booster }\end{array}$ \\
\hline MC 55 & 28/9/12 & 4 site $0.1 \mathrm{ml}$ ID & 0 & 0 & $2.5 \mathrm{IU} / \mathrm{ml}$ \\
\hline MC 58 & 28/9/12 & 4 site $0.1 \mathrm{ml}$ ID & $5 / 10 / 12$ & 2 site 0.1 ml ID (D 7) & $3.5 \mathrm{IU} / \mathrm{ml}$ \\
\hline MC 71 & 28/9/12 & 4 site $0.1 \mathrm{ml}$ ID & $1 / 03 / 13$ & $1 \mathrm{ml}$ I M (6 M) & $4.5 \mathrm{IU} / \mathrm{ml}$ \\
\hline MC 75 & 28/9/12 & 4 site $0.1 \mathrm{ml}$ ID & $10 / 6 / 14$ & 4 site $0.1 \mathrm{ml}$ ID (20 M) & $20 \mathrm{M}+\mathrm{D} 10-3.5 \mathrm{IU} / \mathrm{ml}$ \\
\hline MC 69 & - & Never vaccinated & - & - & $6.5 \mathrm{IU} / \mathrm{ml}$ \\
\hline MC 78 & - & Never vaccinated & - & - & $6.5 \mathrm{IU} / \mathrm{ml}$ \\
\hline MC 104 & 2005 & $2006 \& 20071 \mathrm{ml} \mathrm{IM}$ & $28 / 9 / 10$ & 4 site $0.1 \mathrm{ml} \mathrm{ID}$ & $6.5 \mathrm{IU} / \mathrm{ml}$ \\
\hline
\end{tabular}

$\mathrm{D}=$ Days, $\mathrm{M}=$ Months ${ }^{*}$ after blood samples, all of them were given 4 site IDRV in 2014, but only MC 75 was tested for RIFFIT after 10 days to measure the response of the booster. 
such vulnerable populations like rag pickers as this has a wider margin of safety and is acceptable to such populations. Wider margin of safety is in the sense that if the rag pickers have low immunity due to malnutrition etc. or if the vaccine is low in antigen content or if the vaccinator inadvertently injects the vaccine improperly at any of the site/s. Acceptability is in the sense that the deltoid and suprascapular areas are usually kept exposed in Indian populations compared to other Asian countries.

In our case the antibody response was very good may be because very potent vaccines were used. It is well established that antigen content do matters in intra-dermal vaccination and low dose of antigen would elicit poor response [12]. We, therefore, agree to the demand by Supawant Chutivongse, Henry Wilde et al. [13] that we would like to see the actual potency and antibody content clearly stated on each batch of vaccine and RIGs. This becomes more important in precarious situations, like this, where onetime prophylaxes is to be administered, because some of the prescribed dilutions for vaccine reconstitution are confusing e.g. the vaccine Abhayrab that we also used for 4 site vaccination this year in 2014, supplied as hospital supplies, Batch AYB 17/14, Mft date 2/14, Expr date 1/17 clearly states under the head Reconstitution on the vaccine box of 20 vials that "Reconstitute the freeze dried vaccine with provided $1.0 \mathrm{ml}$ diluent $(0.9 \% \mathrm{w} / \mathrm{v}$ Sodium Chloride Inj. I.P.) for ID administration or reconstitute with $0.5 \mathrm{ml}$ out of $1.0 \mathrm{ml}$ diluents for IM administration”. The vaccine is for Intramuscular/Intradermal Use and licensed in India under Mfg. Lic. No. 15, having potency > $2.5 \mathrm{IU} / \mathrm{vial}$.

\section{Recommendation}

4 site ID anti-rabies vaccination is effective for pre exposure rabies prophylaxis and we recommend the same for special populations like rag pickers and even dog butchers of Nigeria based on our study and also because of the fact that such was a practice in some of the travel clinics in UK in 70's [14] The four sites we used were two deltoid and two supra-scapular regions and not the lower limbs (Thighs) for reasons of acceptability in Indian conditions.

The vaccine companies need to specify the antigen content on their vials for better compliance to precarious situations like this, where one time vaccination is to be done.

\section{Engagement and Dialogue with Rag Pickers}

Engagement and dialogue with rag pickers can help make them understand the value of pre exposure rabies vaccination and combining such an initiative with other health benefits like immunization of their children on spot of their working can boost such a dialogue further. Their engagement in planning for their health initiatives can be another benefit like imparting health and hygiene trainings to garbage collectors and sweepers can help them understand the value of health and hygiene.

For example we also took time to go to them to vaccinate their infants and pregnant mothers on garbage dumps for better dialogue and confidence building with them. We also imparted one day batch-wise training to more than 500 garbage collectors, municipality workers and sweepers on health and hygiene in a reputed venue of the town in 2013. In this training, we practically demonstrated the hand washing technique and also gave a pictorial manual to all of them for better understanding of health related issues including how the garbage can be dangerous if not handled with precaution and what is the concept of environment health and vaccination.

We also organized health check up camps for rag pickers that evoked a good response and prepared them for vaccination. The health insurance camp organized recently was a big hit and helped in better confidence building. We contacted all the scrap dealers to send rag pickers that come to them to the insurance cum vaccination camp as both of them were to be done free.

We are now actively planning to register rag pickers with municipality and also help formalize their rag picking business so that they can get better returns for the garbage they sell to scrap dealers. We are also planning similar campaigns for unorganized labor class in the city who are working as construction workers, kiln workers or those working in small scale industry.

Such initiatives need to be proactively taken by municipal authorities and the municipality needs to own them as their unemployed-unpaid sanitary workforce.

We must not forget that rag pickers are the best environmentalists of the cities, nature and earth!

\section{Limitation}

This study has a limitation of recall bias. However this was largely removed after we retrieved Picture 1 of 


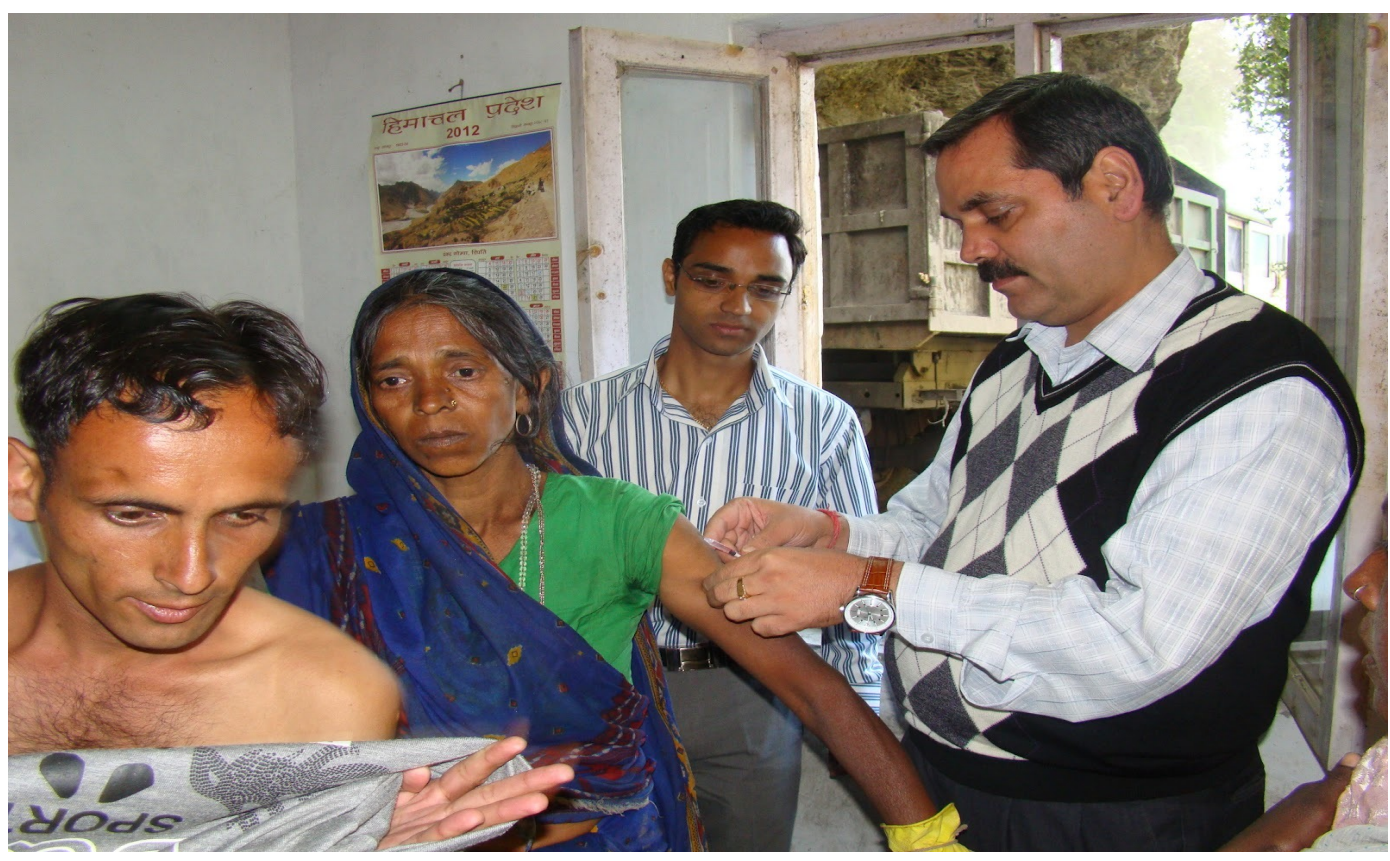

Picture 1. The author vaccinating Rag Pickers at 4 sites in 2012 at one of the municipal garbage dumping sites in the caretaker's shed at Shimla, HP, India.

2012 whereas even we could see 4 site vaccination being done by us but workers who consented for blood samples remembered only 2 site vaccination. Therefore larger studies on volunteers are recommended for 4 site onetime pre exposure rabies vaccination for better understanding of the onetime rabies pre exposure prophylaxis.

\section{Acknowledgements}

We are sincerely thankful to Dr. S.N. Madhusudana for his lab support for doing RFFIT. Also our thanks to Dr. Mary J. Warrell for her active advise on the matter and many thanks to Dr. Henry Wilde for his continuous support with review and literature.

\section{References}

[1] Rauf, M.U.A., Saleem, M.D., Anwer, M.O., et al. (2013) HIV, Hepatitis B and Hepatitis C in Garbage Scavengers of Karachi. Journal of Pakistan Medical Association, 63, 798-802. http://www.jpma.org.pk/PdfDownload/4256.pdf

[2] Khawplod, P., Wilde, H., Benjavongkulchai, M., et al. (2007) Immunogenicity Study of Abbreviated Rabies Preexposure Vaccination Schedules, International Society of Travel Medicine, 1195-1982. Journal of Travel Medicine, 14, 173-176. http://onlinelibrary.wiley.com/doi/10.1111/j.1708-8305.2007.00120.x/pdf http://dx.doi.org/10.1111/j.1708-8305.2007.00120.x

[3] Khawplod, P., Wilde, H., Sriaroon, C., Chomchey, P., Kamolthum, T., Sitprija, V., et al. (2008) One or Three Intradermal Injections within One Week for Rabies Pre-Exposure Immunization. Developments in Biologicals, 131, 393401. http://www.ncbi.nlm.nih.gov/pubmed/18634501

[4] Warrell, M.J. (2012) Current Rabies Vaccines and Prophylaxis Schedules: Preventing Rabies before and after Exposure. Travel Medicine and Infectious Disease, 10, 1-15.

http://www.travelmedicinejournal.com/article/S1477-8939(11)00130-X/abstract http://dx.doi.org/10.1016/j.tmaid.2011.12.005

[5] Bharti, O.K., Damme, W., Decoster, K., Isaakidis, P., Appelmans, A., Ramachandran, V. and Phull, A. (2012) Breaking the Barriers to Access a Low Cost Intra-Dermal Rabies Vaccine through Innovative "Pooling Strategy". World Journal of Vaccines, 2, 121-124. http://dx.doi.org/10.4236/wjv.2012.23016

[6] Garba A., Umoh, J.U., et al. (2015) Rabies Virus Neutralizing Antibodies in Unvaccinated Rabies Occupational Risk Groups in Niger State, Nigeria. International Journal of Tropical Disease \& Health, 6, 35-43. http://dx.doi.org/10.9734/IJTDH/2015/14461

[7] Ekanem, E.E., Eyong, K.I., et al. (2013) Stray Dog Trade Fuelled by Dog Meat Consumption as a Risk Factor for Ra- 
bies Infection in Calabar, Southern Nigeria. African Health Sciences, 13, 1170-1173. http://www.ncbi.nlm.nih.gov/pmc/articles/PMC4056491/ http://dx.doi.org/10.4314/ahs.v13i4.44

[8] Mshelbwala, P.P., et al. (2013) Detection of Rabies Antigen in the Saliva and Brains of Apparently Healthy Dogs Slaughtered for Human Consumption and Its Public Health Implications in Abia State, Nigeria. ISRN Veterinary Science, 2013, Article ID: 468043. http://www.hindawi.com/journals/isrn/2013/468043/

[9] Wertheim, H.F.L., Nguyen, T.Q., Nguyen, K.A.T., de Jong, M.D., Taylor, W.R.J., et al. (2009) Furious Rabies after an Atypical Exposure. PLoS Medicine, 6, e1000044. http://dx.doi.org/10.1371/journal.pmed.1000044

[10] Atuman, Y.J., Adawa, Y.A., Solomon, A., Mshelbwala, P.P. and Ogunkoya, A.B. (2014) Potential Risks for Rabies Spill-Over from Apparently Healthy Dogs to Wildlife in Bauchi State, Nigeria. Journal of Veterinary Advances, 4, 493-498. http://dx.doi.org/10.5455/jva.20140418115203

[11] Mshelbwala, P.P., Ogunkoya, A.B. and Maikai, B.V. (2013) Detection of Rabies Antigen in the Saliva and Brains of Apparently Healthy Dogs Slaughtered for Human Consumption and Its Public Health Implications in Abia State, Nigeria. ISRN Veterinary Science, 2013, Article ID 468043, 5 p. http://dx.doi.org/10.1155/2013/468043.

[12] Dhillon, S., Sampath, K., Moore, C., Dosanjh, A. and Thiel, D. (2013) High-Dose Intra-Dermal Hepatitis B Vaccine in a Liver Transplant Patient Who Failed Prior Intramuscular Vaccination: A Brief Case Report. World Journal of Vaccines, 3, 16-18. http://dx.doi.org/10.4236/wjv.2013.31003

[13] Chutivongse, S., Wild, H., et al. (1991) One-Year Study of the 2-1-1 Intramuscular Postexposure Rabies Vaccine Regimen in 100 Severely Exposed Thai Patients Using Rabies Immune Globulin and Vero Cell Rabies Vaccine. Vaccine, 9, 573-576. http://www.ncbi.nlm.nih.gov/pubmed/1771970 http://dx.doi.org/10.1016/0264-410X(91)90244-Z

[14] Turner, G.S., Aoki, F.Y., et al. (1976) Human Diploid Cell Strain Rabies Vaccine. Rapid Prophylactic Immunization of Volunteers with Small Doses. The Lancet, 307, 1379-1381. http://www.ncbi.nlm.nih.gov/pubmed/59017 http://dx.doi.org/10.1016/S0140-6736(76)93028-2 